\title{
Conditional Monitoring of Switched Reluctance Motor for Static and Dynamic Eccentricity Faults with Fault Isolation
}

\author{
Aduru Veera Reddy*, Balasubramanian Mahesh Kumar \\ Department of Electrical and Electronics Engineering \\ Pondicherry Engineering College, Pondicherry, India \\ *Corresponding author: veerareddyaec@ gmail.com
}

(Received December 17, 2018; Accepted March 21, 2019)

\begin{abstract}
In this paper, the proposed technique interlinking Proteus and Arduino is a novel and cost-effective strategy helps to identify the fault and protect the machine by giving the signal for isolation from supply, it is suitable for all industrial applications. The fault tolerance of the switched reluctance motor is high, but cannot be acclaimed for absolute and should be analyzed under different operating conditions. It is preliminary to examine and diagnose the eccentricity to achieve the smooth running of the motor. The Emulation results for the proposed model successfully exhibited the type of fault, location and also give the details of emergency of repair in a particular section based on the severity of fault in that location. This is a simple and powerful integrated technique feasible to use in all types of laboratory, industrial and research and development applications.
\end{abstract}

Keywords- Eccentricity, Emulator, Isolation, Proteus.

$\begin{array}{ll}\text { Nomenclatures } \\ \mathrm{C}_{\mathrm{s}} & \text { Stator center } \\ \mathrm{C}_{\mathrm{R}} & \text { Rotor center } \\ \mathrm{C}_{\mathrm{W}} & \text { Rotation center } \\ \mathrm{d} & \text { Air gap length under fault condition in } \mathrm{mm} \\ \mathrm{g} & \text { Uniform air gap length in } \mathrm{mm} \\ \mathcal{E} & \text { Eccentricity }\end{array}$

$\begin{array}{ll}\text { Abbreviations } \\ \text { DE } & \text { Dynamic Eccentricity } \\ \text { FEM } & \text { Finite Element Method } \\ \text { SE } & \text { Static Eccentricity } \\ \text { SRM } & \text { Switched Reluctance Motor } \\ \text { ME } & \text { Mixed Eccentricity }\end{array}$

\section{Introduction}

The switched reluctance motor (SRM) has found numerous applications in various sectors due to its simple structure and robust build. The mathematical modeling, the design of an aerial vehicle to obtain its structural characteristics using numerical methods like Finite Element Method (FEM) is essential (Raj et al., 2019). The most common faults that a motor can experience is an air-gap eccentricity. It may be because of the mechanical problems or due to the defects in the electrical or magnetic system. The non-uniform air gap effect on the overall static torque by observing Flux density harmonics and harmonic torque variation in the cases of eccentricity presented by Sheth and Rajagopal (2005). Design and optimization of B-pillar reinforcement using finite element analysis for better performance of automobile applications demonstrated by Ikpe et al., (2017). 
International Journal of Mathematical, Engineering and Management Sciences

Vol. 4, No. 3, 671-682, 2019

https://dx.doi.org/10.33889/IJMEMS.2019.4.3-053

Lee et al. (2016), employed the online current signature analysis to identify the false rotor faults. According to (Gunji et al., 2019), the computer-aided design based algorithm used to identify the clash information in robotic assembling with respect to various industrial and domestic applications. The method used by Faiz et al. (2009) employs the signal spectra of stator currents and evaluated sidebands to study SE, ME in induction motor by employing a Modified Winding Function method. With reference to Li et al. (2009), the external winding method compensated the effect of eccentricity on electrical and magnetic parameters in 12/8 switched reluctance motor.

Hong et al. (2012) illustrated the FE simulation and experimental study of 10-hp PMSM for healthy and different air gap eccentricities detected with the help of variation of d-axis equivalent inductance. The stress analysis of flat plates with various geometrical parameter cut-outs by considering unique and bi-axial loading ratios using ANSYS software tools have elucidated by Gunwant (2019). Drif and Cardoso (2008), Yahia et al. (2016) investigated the occurrence of air gap faults in induction motor using modified pony's method. Singh and Kumar (2017), employed the stator current control for bearing fault diagnosis in mechanical systems. In BLDC motor, Park and Hur (2016) adopted the Fast Fourier Transform to analyze fault diagnosis in rotating machines and investigated the inter-turn and dynamic eccentricity using frequency spectrum. The polarity having an impact on the strength of arc welding of steel with different structural and mechanical properties (Khamari et al., 2019). Arduino based black box model design for monitoring the accidental details of vehicles (Pasam et al., 2016).

This method has not appeared before, most of the papers deal with only off-line or no load cases, the proposed method considering the full load condition. The objective of this work is to identify the fault type, noticing its reaction in different locations and isolate the machine from operation if a fault is more severe. A 2-Dimensional finite element model has been employed to construct a normal motor and motor with a misaligned rotor. This fault will result in serious damage to the motor stator or rotor core. In this paper, Section 2 deals with the FEM Model, The main eccentricity faults occurring in SRM are presented in Section 3. The sample SRM characteristics are obtained by 2D-FEM analysis, the comparison of simulation results for various types of eccentricities being presented and discussed in Section 4. The final conclusions are reported in Section 5. The proposed method is a simple and novel method useful in real-time applications like automatic detection of faults in industrial applications.

\section{FEM Modeling of Switched Reluctance Motor for Normal and Eccentricity Faulty Cases}

\subsection{Normal Motor}

The 2-D FEM Modeling of Switched reluctance motor under the healthy condition is shown in Figure 1. In the case of $8 / 6$ pole fault free motor the rotor typically is placed at the center position of the stator bore and appears in the same air-gap between them. Under such normal condition, the balanced magnetic forces are acting in the counter directions. The direction of torque is always towards the aligned position, the instantaneous torque is

$$
\mathrm{T}=\frac{1}{2} i^{2} \frac{\partial L}{\partial \theta}
$$

The stator phase current has to be turned on during raising inductance portion to obtain positive torque in motoring action. Since the current constant, torque waveform follow the same tendency of inductance profile of the motor. 
International Journal of Mathematical, Engineering and Management Sciences

Vol. 4, No. 3, 671-682, 2019

https://dx.doi.org/10.33889/IJMEMS.2019.4.3-053

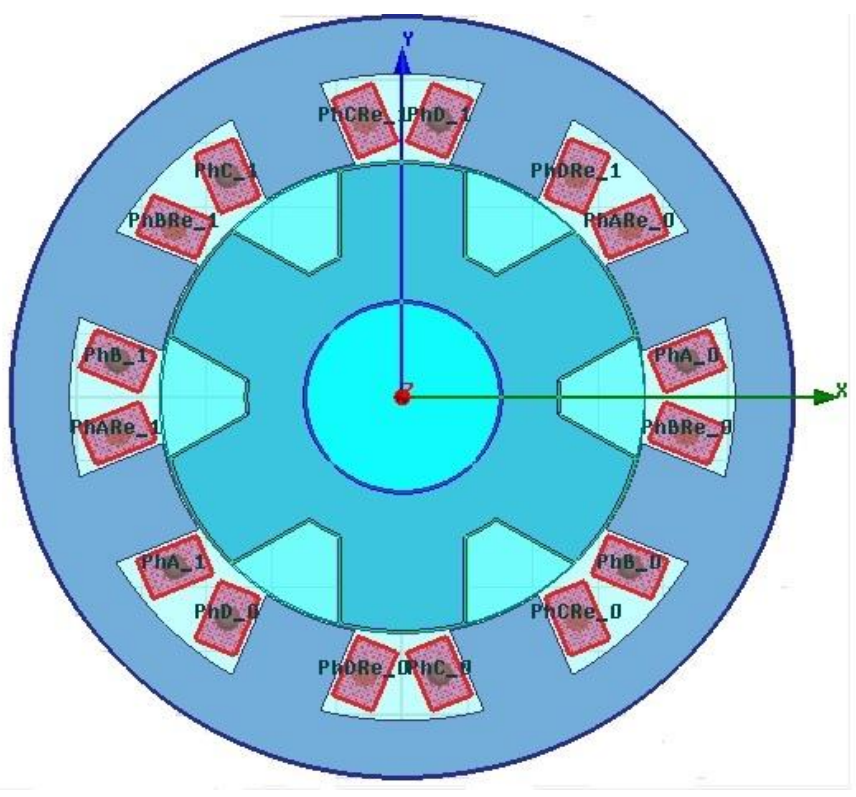

Figure 1. Switched reluctance motor 2D model

There is a reduction in air gap on one side, and subsequent increase in air gap on the other side during eccentricity condition. This variation causes larger absorption inattention force in the shorter gap side. Due to the severe operating environment, there is likelihood that the rotor will be dislocated from its center point.

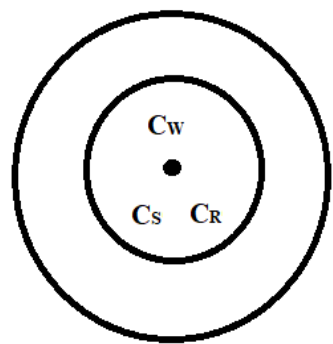

a) Normal

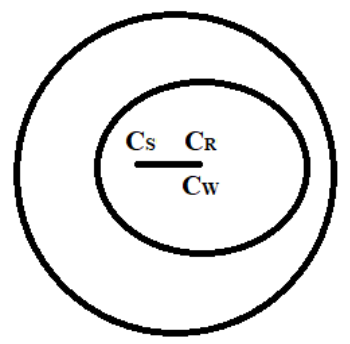

b) Static eccentricity

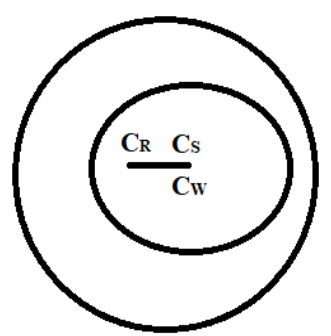

c) Dynamic eccentricity

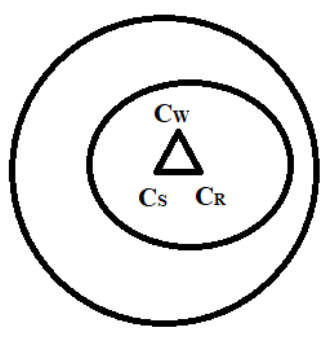

d) Mixed eccentricity

Figure 2. Motor under eccentricity

The displacement can happen because of abnormal conditions within the machine. Based on the coincidence of stator center $C_{s}$, rotor center $C_{R}$ and rotation center $C_{w}$, the three types of eccentricities static, dynamic and mixed eccentricities and its geometrical representation as shown in Figure 2.

\subsection{Static Eccentricity}

The static eccentricity can be arrived owing to a number of reasons like elliptical stator core, incorrect placement of rotor or stator during assembling and dismantling, error during the 
maintenance, improper placing or breakage of bearings. Faiz and Pakdelian (2008) explained the non-uniformity of flux in air gap results in huge unbalanced forces in the motor. This force pull the rotor in such a way that, it moves in the direction of the lower air gap side as shown in Figure 3 . This subsequently produces heavy noise and vibrations, it may lead to severe damage to the various internal and external parts of the motor.

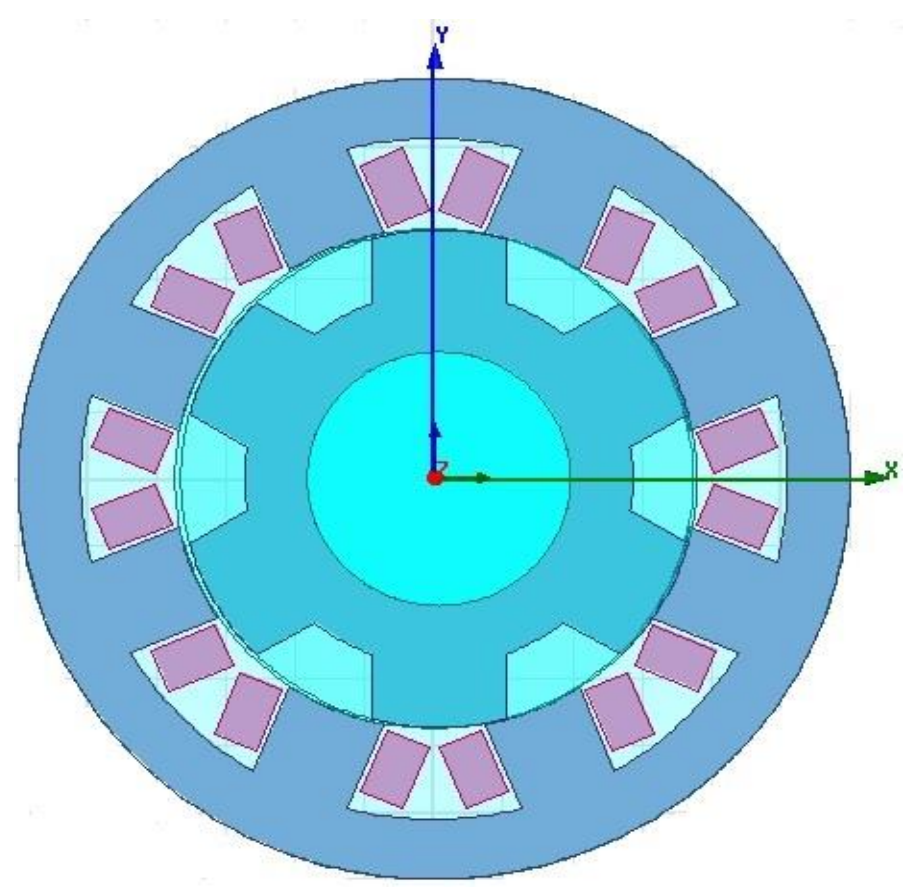

Figure 3. Motor under eccentricity fault

\subsection{Dynamic Eccentricity}

When the position of stator axis and rotation axis centers is coincident but displaced with reference to the rotor is known as dynamic eccentricity. It occurs if any problem in the bearings, bent of a shaft or could be produced by manufacturing ignorance. The path of rotor center traces an elliptical orbit about the stator bore axis. If it ignores, it may cause for physical rubbing of stationary and rotating parts, results in extensive damage of motor.

\subsection{Mixed Eccentricity}

In some cases, both SE and DE have simultaneously happened then it is called Mixed Eccentricity. This occurs due to the failure of bearings. The fault level can be expressed in terms of eccentricity percentage.

$\% \varepsilon=\left(\frac{d}{g}\right) \times 100$ 
International Journal of Mathematical, Engineering and Management Sciences

Vol. 4, No. 3, 671-682, 2019

https://dx.doi.org/10.33889/IJMEMS.2019.4.3-053

where $\varepsilon$ is the eccentricity, $g$ is the length of normal air gap, $d$ is the change in air gap under fault case. If $d$ is more then it leads to very high-level eccentricity faults, impacts the machine with unpredictable effects like unbalanced forces, noise, overheating and excessive vibrations.

\section{Analysis of Stator Current under Normal and Eccentricity Faulty Cases}

In this work three different motor configurations are analyzed, one is the normal and other two cases under static, dynamic eccentricity fault conditions up to the fault level of $44 \%$ with different possible combinations. In fault free motor all most all the phase currents having the same values of 6.9A as shown in Figure 4. The static eccentricity fault is designed and interpreted for various scales of eccentricity namely $10 \%, 30 \%$, and $44 \%$. The currents in different phases of a motor under this fault are slightly increased as compared to normal motor shown in Figure 5.

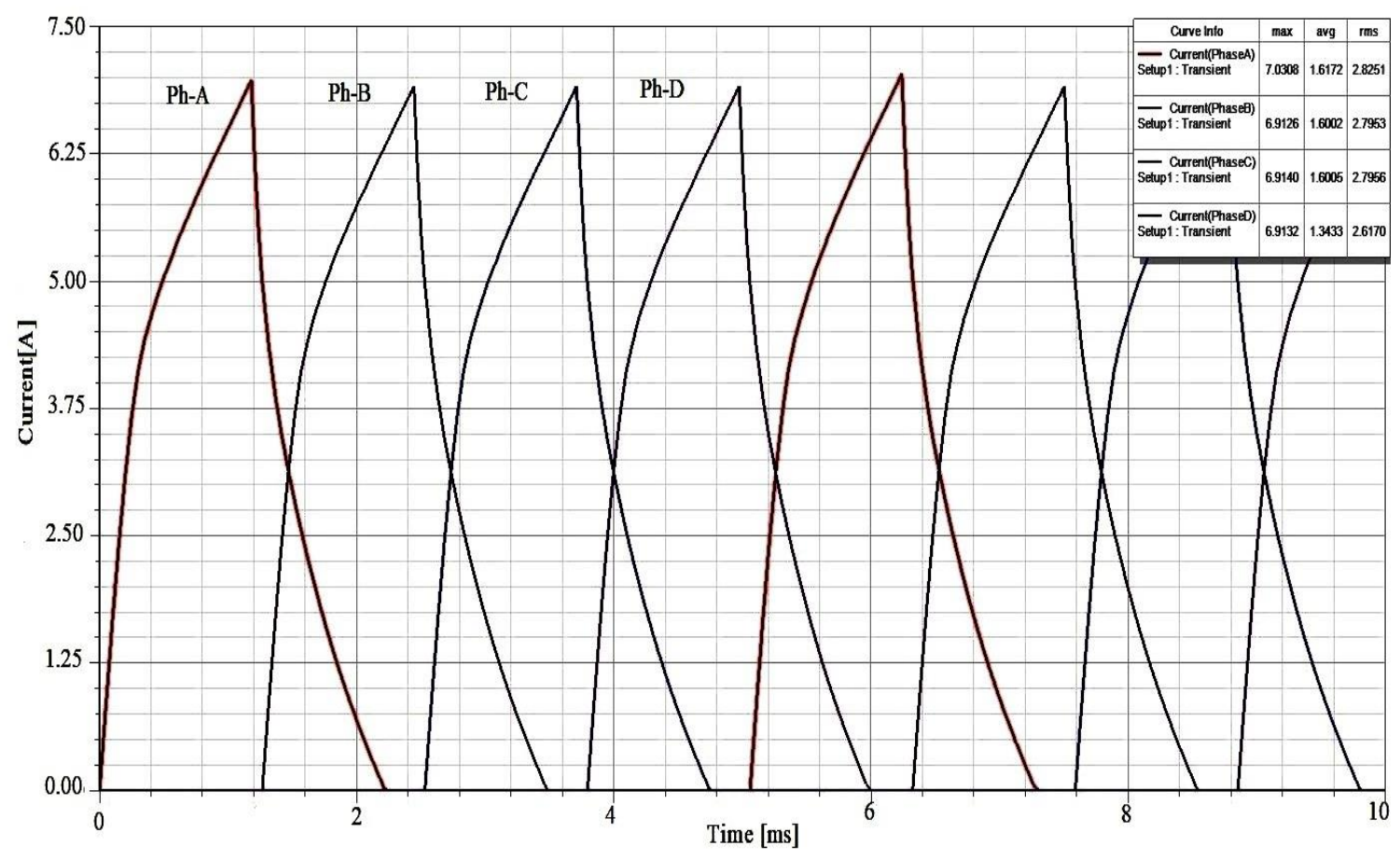

Figure 4.Current wave forms of motor in good condition 
International Journal of Mathematical, Engineering and Management Sciences

Vol. 4, No. 3, 671-682, 2019

https://dx.doi.org/10.33889/IJMEMS.2019.4.3-053

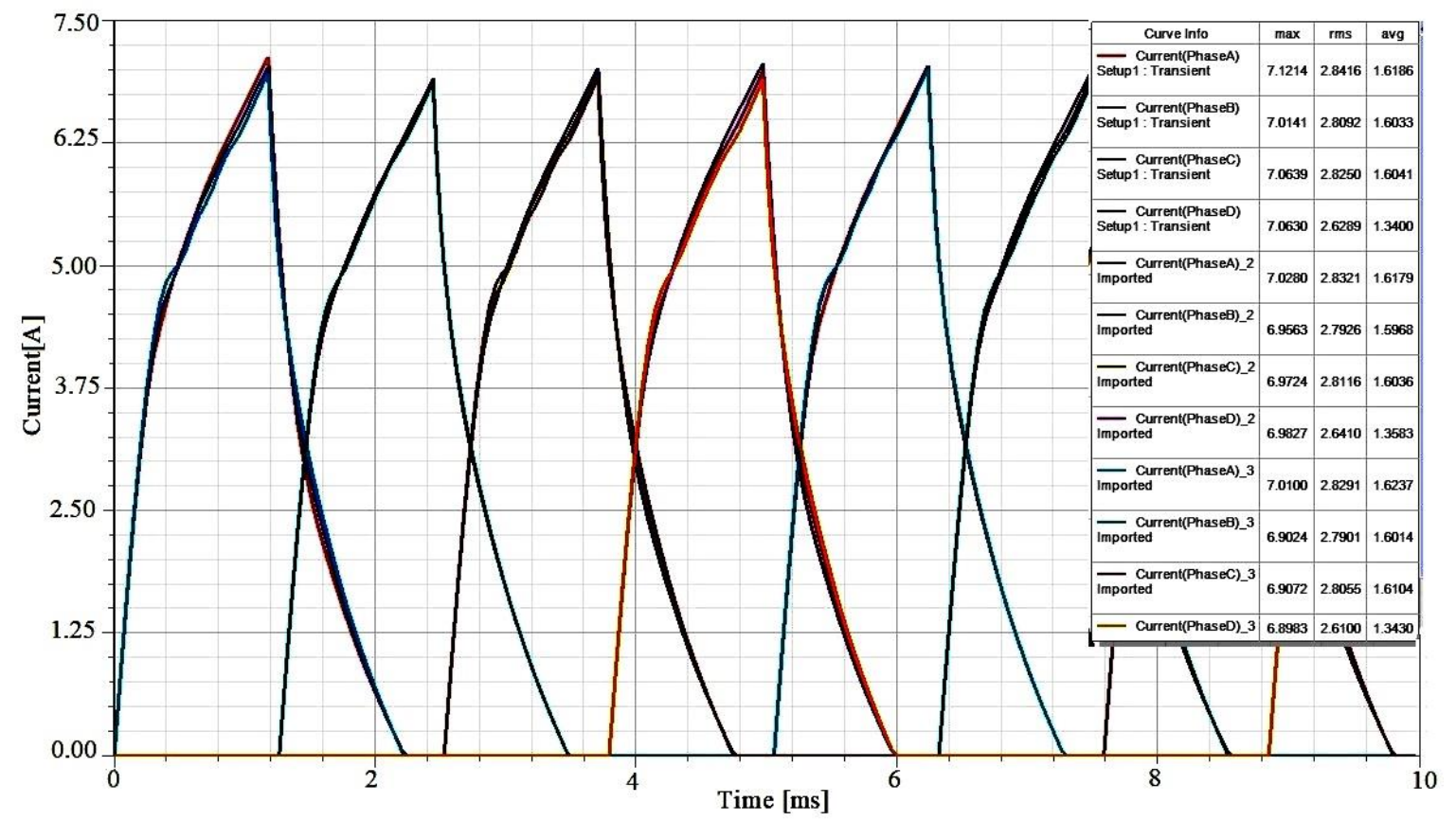

Figure 5. Current wave forms of 10\%, 30\%, $44 \%$ SE

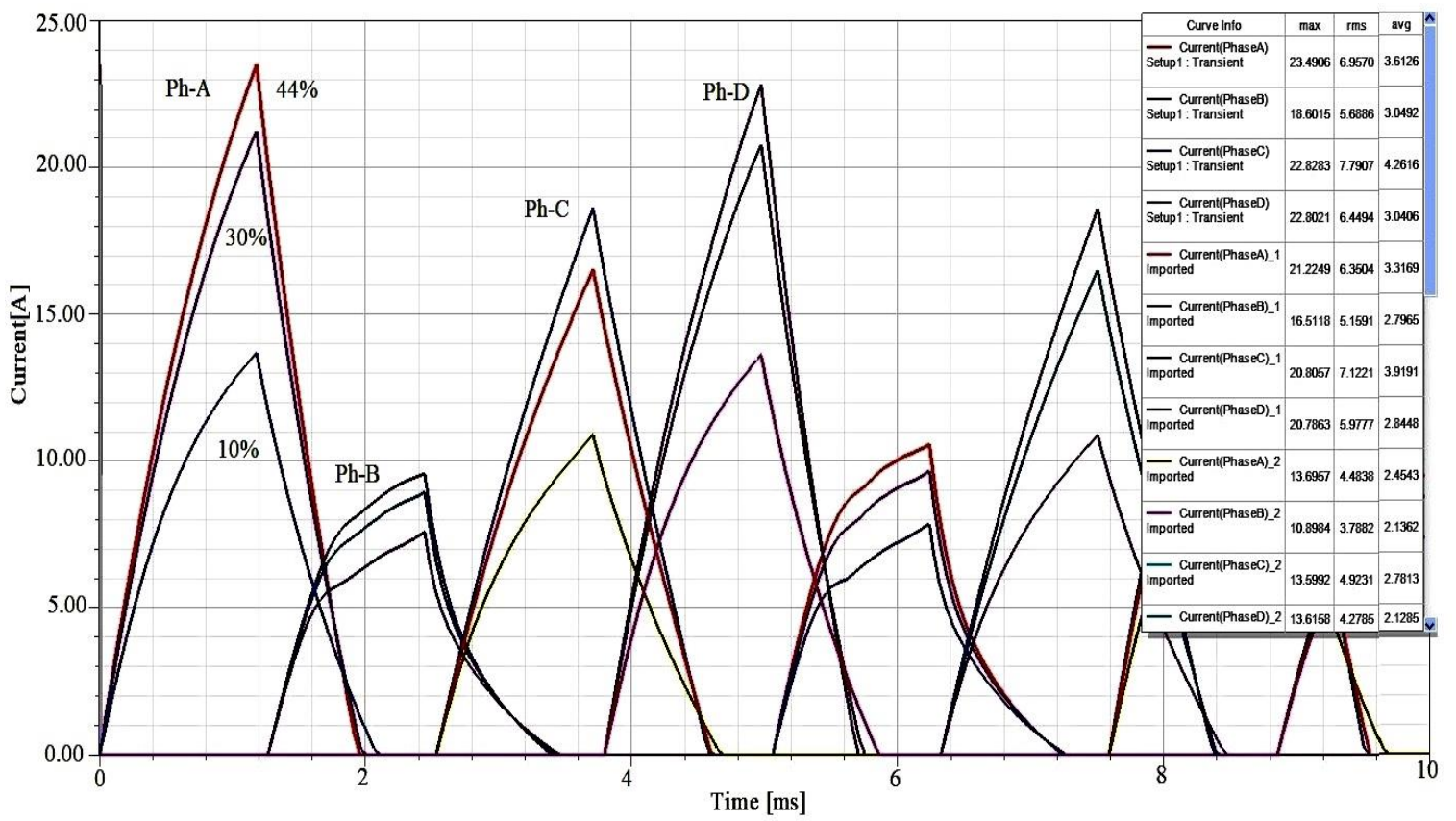

Figure 6. Current wave forms of $10 \%, 30 \%, 44 \%$ DE 
International Journal of Mathematical, Engineering and Management Sciences

Vol. 4, No. 3, 671-682, 2019

https://dx.doi.org/10.33889/IJMEMS.2019.4.3-053

In these cases, the variations are less due to the fixed minimal radial length of an air gap. Average current increases due to the increasing divergence of the current or flux linkages at the higher degree of eccentricity.

In dynamic eccentricity fault, the currents in affected phases are abnormal. The four phases having different current values due to the distinct air gaps caused under poles. This causes an uneven flux distribution around the stator and leads to heavy unbalanced magnetic forces acting on the rotor, huge vibrations and noise occur in the machine when it is running. The current waveforms for various degrees of eccentricity are shown in Figure 6. It can be noted that the peak values of currents in different phases for normal and faulty cases happened at the same moment.

\section{Proposed Method for Fault Detection}

Automatic fault finding and protection has always been a challenging task when monitoring any rotating machinery. Fault diagnosis in machines is a part of mechanical engineering concerned with finding emerging faults. If the state of the machines being used isn't observed the subsequent reactions may cause huge economic and safety problems. Thesummarizeddifferential currents comparison for different conditions given in Table 1 and Table 2. The different cases of severities are considered as LOW, MED (Medium) and HIG (High)

Table 1 Static eccentricity

\begin{tabular}{|c|c|c|c|c|}
\hline \multirow{2}{*}{ Eccentricity } & \multicolumn{4}{|c|}{$\begin{array}{c}\text { Differential Currents in } \\
\text { Phases in Static eccentricity }\end{array}$} \\
\cline { 2 - 5 } & $\Delta \mathrm{I}_{\mathrm{a}}(\mathrm{A})$ & $\Delta \mathrm{I}_{\mathrm{b}}(\mathrm{A})$ & $\Delta \mathrm{I}_{\mathrm{c}}(\mathrm{A})$ & $\Delta \mathrm{I}_{\mathrm{d}}(\mathrm{A})$ \\
\hline $0 \%$ & 0 & 0 & 0 & 0 \\
\hline $10 \%$ & 0.110 & 0.020 & 0.053 & 0.063 \\
\hline $30 \%$ & 0.128 & 0.056 & 0.072 & 0.082 \\
\hline $44 \%$ & 0.208 & 0.125 & 0.155 & 0.182 \\
\hline
\end{tabular}

Table 2 Dynamic eccentricity

\begin{tabular}{|c|c|c|c|c|}
\hline \multirow{2}{*}{ Eccentricity } & \multicolumn{4}{|c|}{$\begin{array}{c}\text { Differential Currents in } \\
\text { Phases Dynamic eccentricity }\end{array}$} \\
\cline { 2 - 5 } & $\Delta \mathrm{I} \mathrm{a}(\mathrm{A})$ & $\Delta \mathrm{I} b(\mathrm{~A})$ & $\Delta \mathrm{I}(\mathrm{A})$ & $\Delta \mathrm{I}(\mathrm{A})$ \\
\hline $0 \%$ & 0 & 0 & 0 & 0 \\
\hline $10 \%$ & 6.8 & 0.6 & 4.1 & 13.8 \\
\hline $30 \%$ & 14.3 & 2 & 9.6 & 15.9 \\
\hline $44 \%$ & 16.7 & 2.6 & 11.7 & 0.6 \\
\hline
\end{tabular}


International Journal of Mathematical, Engineering and Management Sciences

Vol. 4, No. 3, 671-682, 2019

https://dx.doi.org/10.33889/IJMEMS.2019.4.3-053

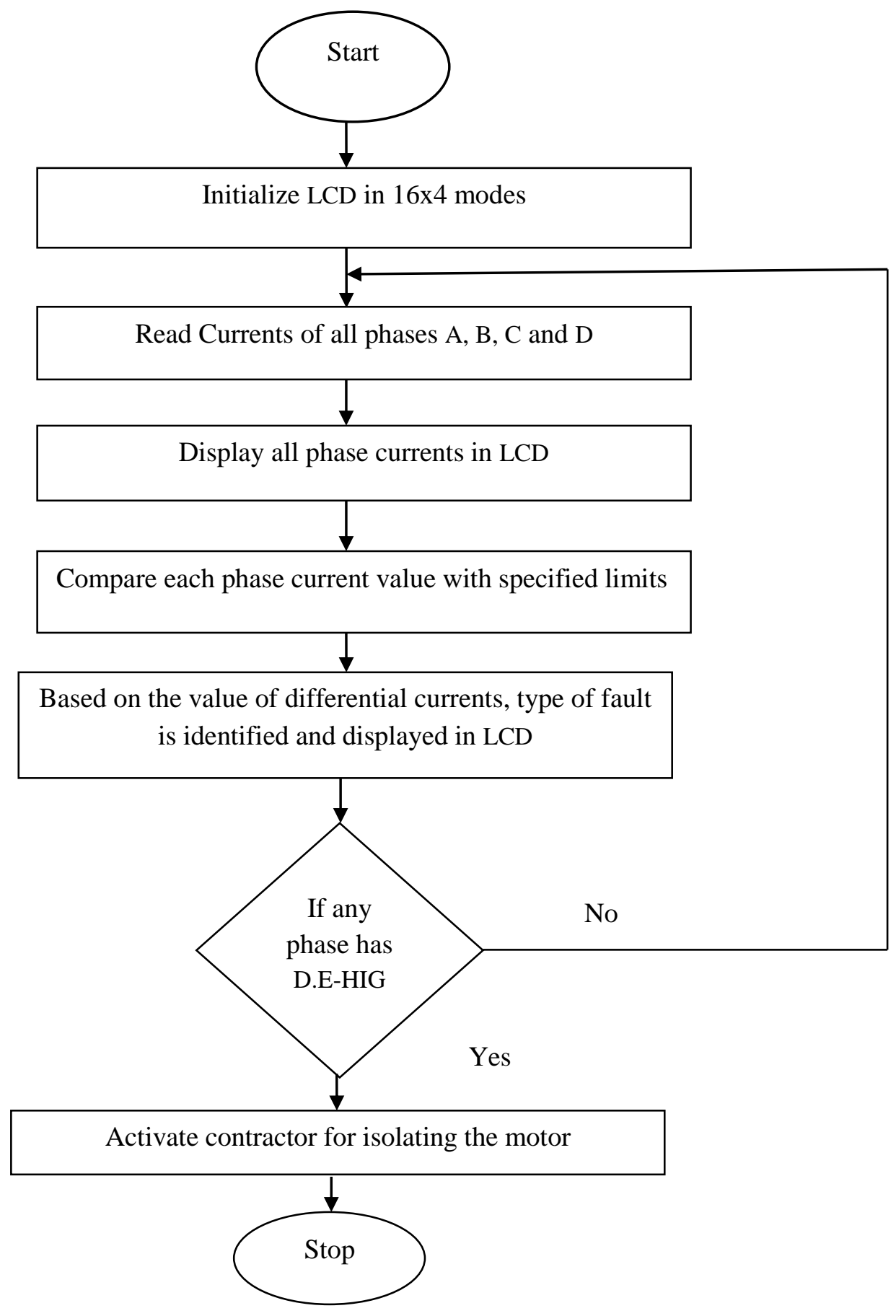

Figure 7. Flow chart for fault identification 
International Journal of Mathematical, Engineering and Management Sciences

Vol. 4, No. 3, 671-682, 2019

https://dx.doi.org/10.33889/IJMEMS.2019.4.3-053

The flow chart is shown in Figure 7 executes the condition of machine time to time-based on our requirement.It directly displays the type of fault and its severity. The proposed method using the Proteus as emulator and Arduino as a simulator.In order to emulate the hardware controller used is ATMega328P. Figure 8 shows the healthy condition, Figure 9 shows the static eccentricity and Figure 10 shows the dynamic eccentricity condition of the motor.

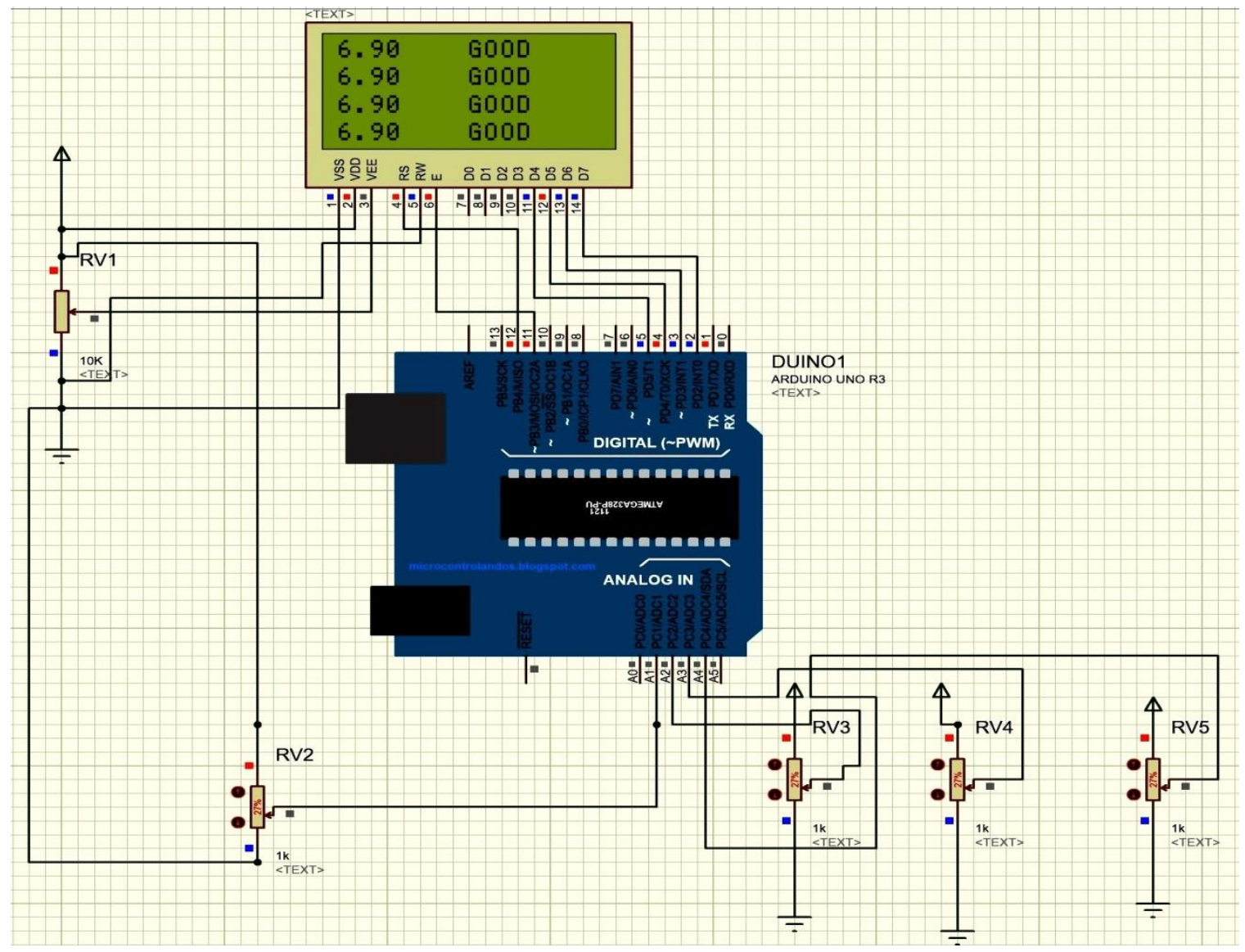

Figure 8. Motor under good condition

According to the range of differential currents, the condition of the machine may be displayed as GOOD,S.E (Static Eccentricity), D.E(Dynamic Eccentricity). The LCD displays the faulty condition and location of fault. It also gives the information about the severity of fault like LOW, MED (Medium), HIG (High). Based on the level of fault it may give an indication ,if particularly in D.E-HIG case it gives a trip signal to a contractor to isolate the motor since it is more dangerous than all the cases. In low and medium cases it gives a warning to be causious to contionue in the same situation and managing the continity of service with out any harm to the machine. 
International Journal of Mathematical, Engineering and Management Sciences

Vol. 4, No. 3, 671-682, 2019

https://dx.doi.org/10.33889/IJMEMS.2019.4.3-053

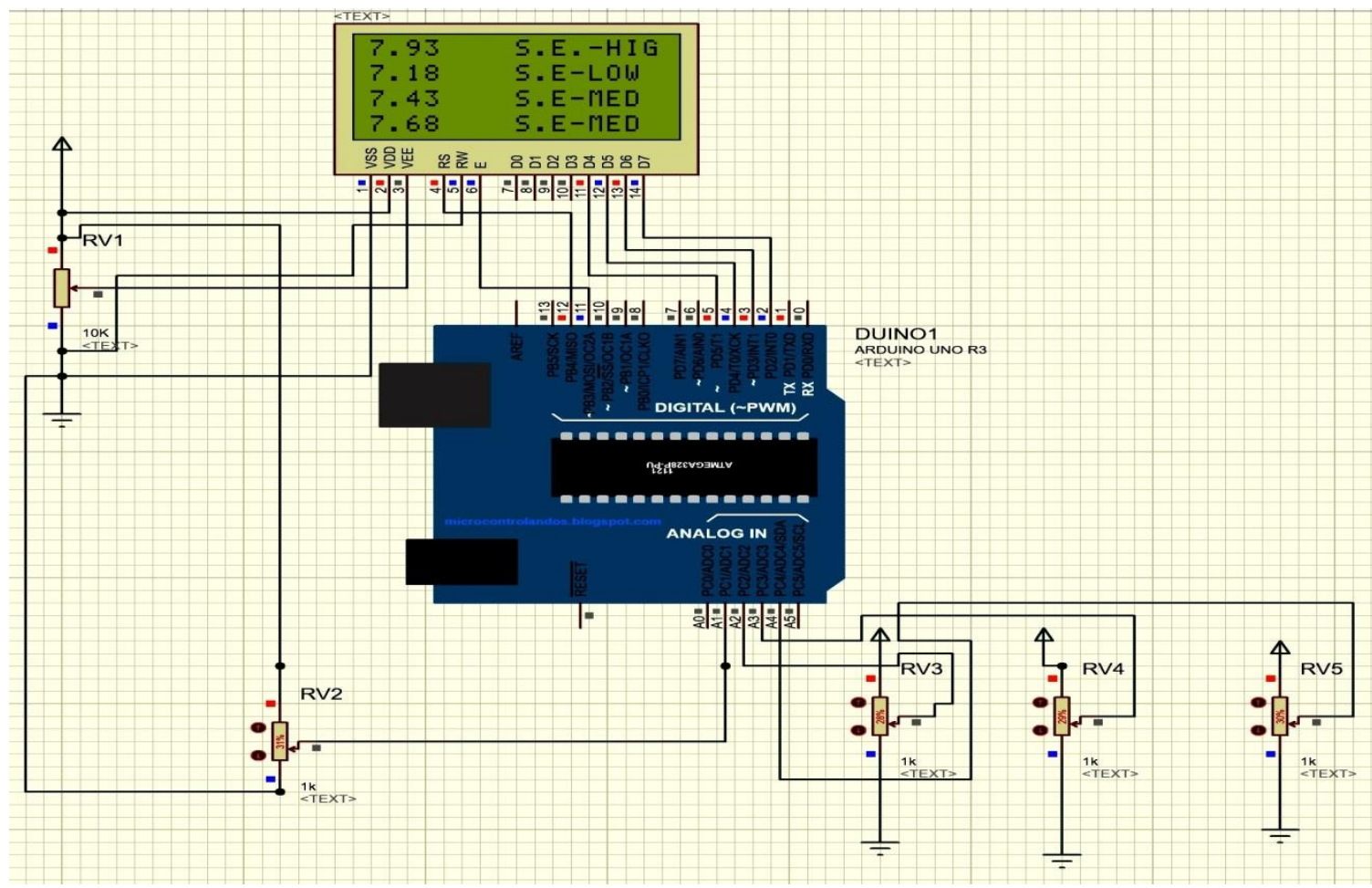

Figure 9. Motor experiencing static eccentricity fault

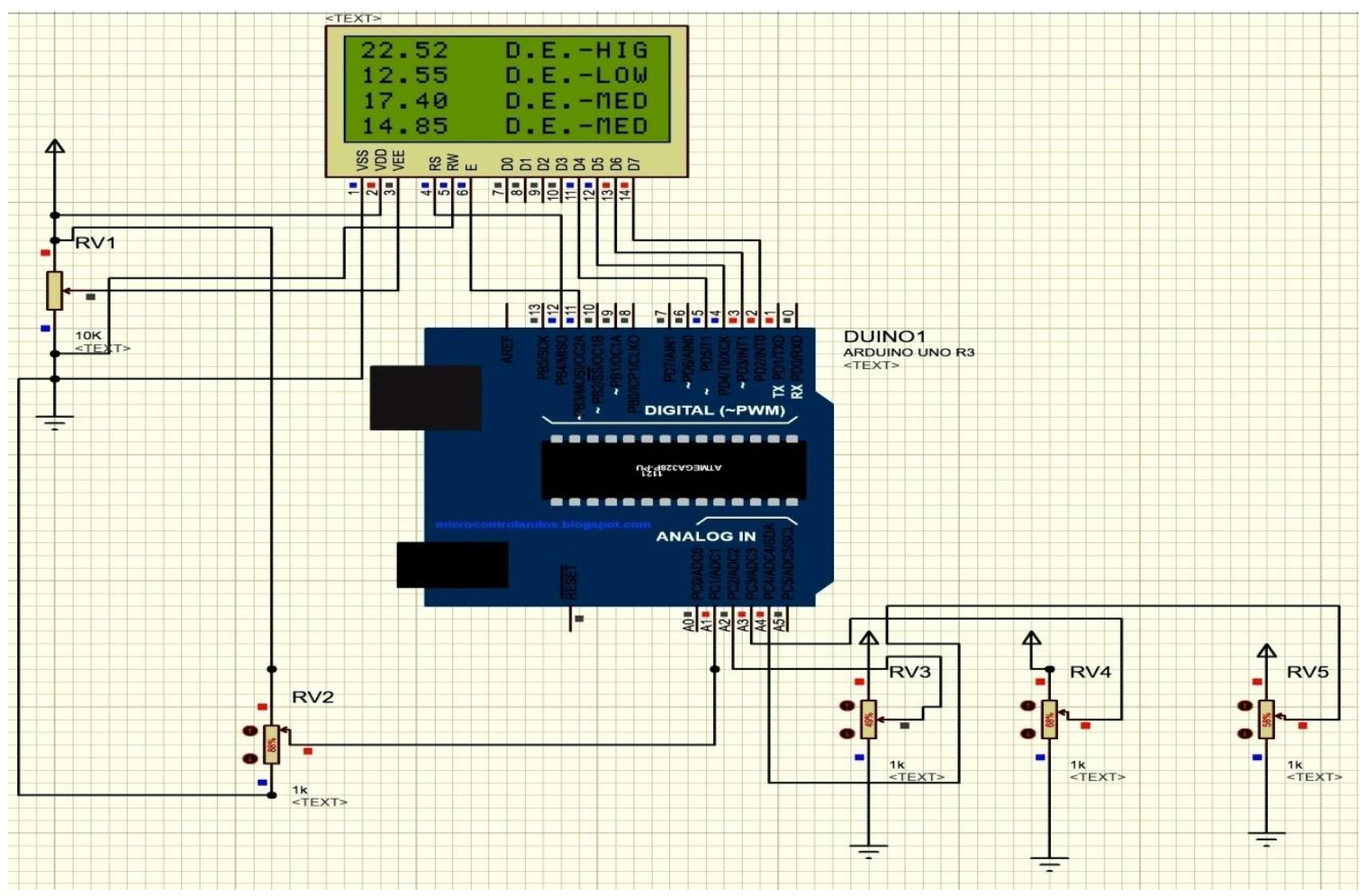

Figure 10. Motor experiencing dynamic eccentricity fault 
International Journal of Mathematical, Engineering and Management Sciences

Vol. 4, No. 3, 671-682, 2019

https://dx.doi.org/10.33889/IJMEMS.2019.4.3-053

\section{Conclusions}

The effect of SE and DE on crest value of currents in an 8/6 SRM are elucidated for distinct degrees of eccentricities $10 \%, 30 \%, 44 \%$ under full load condition. The proposed technique receives the differential currents of a stator winding of motor as input, automatically detects the fault type and its severity. It allows the maximum flexibility of identifying the faults even in a very narrow margin. If the fault is more severe then it isolates the machine from operation using contractor in hardware, so that its saves economy, manpower, and continuity of service. This method can be extended in a generalized way to know any abnormal conditions. This is a costeffective method useful even in very small scale industries.

\section{Conflict of Interest}

The authors confirm that there is no conflict of interest to publish the paper in the journal.

\section{Acknowledgement}

The author would like to express sincere thanks to the referees for their valuable suggestions towards the improvement of the paper.

\section{References}

Raj, P.G., Esakki , B., \& Ganesan, S. (2019). Evaluation of mechanical strength characteristics of double ducted unmanned amphibious aerial vehicle using finite element analysis. International Journal of Mathematical, Engineering and Management Sciences, 4(2), 420-431.

Drif, M.H., \& Cardoso, A.M. (2008). Airgap-eccentricity fault diagnosis, in three-phase induction motors, by the complex apparent power signature analysis. IEEE Transactions on Industrial Electronics, 55(3), 1404-1410.

Gunji, B.M., Deepak, B.B.V.L., Khamari, B.K., \& Biswal, B.B. (2019). CAD-based automatic clash analysis for robotic assembly. International Journal of Mathematical, Engineering and Management Sciences, 4(2), 432-441.

Faiz, J., Ebrahimi, B.M., \& Toliyat, H.A. (2009). Effect of magnetic saturation on static and mixed eccentricity fault diagnosis in induction motor. IEEE Transactions on Magnetics, 45(8), 3137-3144.

Faiz, J., \& Pakdelian, S. (2008). Diagnosis of static eccentricity in switched reluctance motors based on mutually induced voltages. IEEE Transactions on Magnetics, 44(8), 2029-2034.

Hong,J., Lee, S.B., Kral, C., \& Haumer, A. (2012). Detection of airgap eccentricity for permanent magnet synchronous motors based on the d-axis inductance. IEEE Transactions on Power Electronics, 27(5), 2605-2612.

Gunwant, D. (2019). Stress concentration studies in flat plates with rectangular cut-outs using finite element method. International Journal of Mathematical, Engineering and Management Sciences, 4(1), 66-76.

Lee, S.B., Hyun, D., Kang, T.J., Yang, C., Shin, S., Kim, H., Park, S., Kong, T., \& Kim, H.D. (2016) identification of false rotor fault indications produced by online MCSA for medium-voltage induction machines. IEEE Transactions on Industrial Applications, 52(1), 729-739.

Li, J., Choi, D., \& Cho,Y. (2009). Analysis of rotor eccentricity in switched reluctance motor with parallel winding using FEM. IEEE Transactions on Magnetics, 45(6), 2851-2854. 
International Journal of Mathematical, Engineering and Management Sciences

Vol. 4, No. 3, 671-682, 2019

https://dx.doi.org/10.33889/IJMEMS.2019.4.3-053

Ikpe, A.E., Orhorhoro, E.K., \& Gobir, A. (2017). Design and reinforcement of a b-pillar for occupants safety in conventional vehicle applications. International Journal of Mathematical, Engineering and Management Sciences, 2(1), 37-52.

Park, J.K., \& Hur, J. (2016). Detection of inter-turn and dynamic eccentricity faults using stator current frequency pattern in IPM-type BLDC motors. IEEE Transactions on Industrial Electronics, 63(3), 1771-1780.

Sheth, N.K., \& Rajagopal, K.R. (2005). Variations in overall developed torque of a switched reluctance motor with air gap nonuniformity. IEEE Transactions on Magnetics, 41(10), 3973-3975.

Singh, S., \& Kumar, N. (2017). Detection of bearing faults in mechanical systems using stator current monitoring . IEEE Transactions on Industrial Informatics, 13(3), 1341-1349.

Khamari, B.K., Gunji, B., Karak, S.K., \& Biswal, B.B. (2019). Variation of microstructural and mechanical properties with respect to polarity in shielded metal arc welding of mild steel. International Journal of Mathematical, Engineering and Management Sciences, 4(2), 521-530.

Yahia, K., Sahraoui, M., Cardoso, A.J.M.., \& Ghoggal, A. (2016). The use of a modified Prony's method to detect the airgap eccentricity occurrence in induction motors. IEEE Transaction on Industrial Applications, 52(5), 3869-3877.

Pasam, G.K., Ghnimi, S.A., Maskari, A.N.K.A., \& Habsi, Z.A.O.A. (2016). Development of a black box model for the monitoring of vehicle accident and speed. International Journal of Engineering Applied Sciences and Technology, 1(9), 124-130.

(c) (i) Original content of this work is copyright ( ) International Journal of Mathematical, Engineering and Management Sciences. All rights reserved Except of uses under a Creative Commons Attribution 4.0 International (CC BY 4.0) license at https://creativecommons.org/licenses/by/4.0/ 